\title{
Energy-Efficient Routing in Ad Hoc Networks Relying on Channel State Information and Limited MAC Retransmissions
}

\author{
Jing Zuo, Chen Dong, Soon Xin Ng, Lie-Liang Yang and Lajos Hanzo \\ School of ECS, University of Southampton, SO17 1BJ, UK. \\ Tel: +44-23-8059 3125, Fax: +44-23-8059 4508 \\ Email: \{jz08r,cd2g09,sxn,lly,lh\}@ecs.soton.ac.uk; http://www-mobile.ecs.soton.ac.uk
}

\begin{abstract}
In ad hoc networks the nodes actively and voluntarily participate in constructing a network and act as relays for other nodes. As a result of node-mobility, the Channel State Information (CSI) varies and hence a substantial amount of control messages have to be exchanged across the network to maintain reliable communications between certain pairs of nodes, which potentially imposes a high energy-consumption. Therefore, minimizing the energy consumption and maximizing the throughput of ad hoc nodes is extremely important. This paper analyzes both the energy consumption and the achievable throughput of a multihop route by exploiting both the CSI quantified, for example in terms of the Frame Error Ratio (FER), as well as the number of Medium Access Control (MAC) retransmissions and the number of hops. Since using limited number of MAC retransmissions in a hop-by-hop retransmission mode imposes dependencies amongst the links of a multi-hop route, a more accurate objective function may be formulated than that which assumes the availability of an infinite number of retransmissions and which considers the links to be independent. Our simulations confirm the improved accuracy of the proposed Objective Function (OF), especially when the FER and the number of hops are high and the number of MAC retransmissions is low. Additionally, a low-complexity routing algorithm is designed, which carries out routing decisions based on the energy consumption predicted by the $\mathrm{OF}$, and strikes a compromise between having 'few long-distance hops' and 'many short-distance hops' for the sake of energy minimization and throughput maximization.
\end{abstract}

\section{INTRODUCTION}

In recent years, numerous energy-efficient techniques have been proposed [1-6]. However, simply minimizing the energy consumption results in deficient designs. It is more beneficial to strike a tradeoff between the energy consumed and other metrics, such as the attainable throughput. For example, a Minimum-Power Cooperative Routing (MPCR) algorithm was proposed in [1], which identifies and constructs the minimum-power route without unduly compromising the throughput. Multiple-Input and Multiple-Output (MIMO) schemes and near-capacity Space-Time Codes (STCs) were employed in [2] for optimizing the relay selection for the sake of maximizing the end-to-end throughput at a given total available power. While single-hop transmissions are more suitable for bandwidth-limited scenarios, multi-hop transmissions combined with spatial frequencyreuse tend to perform better in power-limited situations [3]. Spatial frequency-reuse employed in multi-hop scenarios may be beneficially combined with Interference Mitigation (IM) $[2,3]$ and transmit beamforming [2] for the sake of finding an attractive balance between energy minimization and throughput maximization in both singlehop and multi-hop schemes [3-5]. As a further advance, the authors of $[4,6]$ jointly considered both the transmit-energy and the signalprocessing-related circuit-energy consumption. A beneficial tradeoff between the total energy consumption and throughput was found in [4] by considering both the transmission strategy of each node as well as the location of the relay nodes and the data rate of each node. In [6], a convex optimization technique was used for tackling the cross-layer energy optimization problem. It combined single-hop and multi-hop transmissions, with the conclusion that circuit energy minimization favored transmissions relying on fewer hops.

${ }^{1}$ The research leading to these results has received funding from the European Union's Seventh Framework Programme (FP7/2007-2013) under grant agreement no 214625. The financial support of the China-UK Scholarship Council, and of the RC-UK under the auspices of the IU-ATC initiative is also gratefully acknowledged.
The physical layer, Medium Access Control (MAC) layer, network layer and even the transport layer often cooperate with each other for the sake of energy minimization. For example, the impact of the link error rate on the route selection between a path associated with a large number of short-distance hops and another with a smaller number of long-distance hops was studied in [5]. In this paper, the link 'cost' was defined as a function of both the energy required for a single transmission attempt and the link error rate. This Objective Function (OF) captures the cumulative energy expended in reliable data transfer for both reliable and unreliable link layers. In [7], several routing algorithms were proposed, which opted for the route with minimum energy consumption in a mixed hop-by-hop and end-to-end retransmission mode. In the end-to-end retransmission mode, a single unreliable link may require retransmissions from the source, and hence may require more energy for successfully delivering packets. Consequently, routing protocols play an important role in saving energy. The authors of [8] took into account both the energy consumed by data packets as well as by control packets and MAC retransmissions, because ignoring the energy consumption of exchanging control packets might underestimate the actual energy consumption and thus may lead to inefficient designs. Furthermore, energy-efficient unicast and multicast routing protocols were proposed in [9], which relied on diverse power control techniques.

However, the energy OFs employed in $[5,7,8]$ exploited the assumption of having access to a potentially infinite number of MAC retransmissions, which is unrealistic. Additionally, although the authors of [9] considered a limited number of MAC retransmissions, no specific OF was formulated. Against this backdrop, the novel contributions of this treatise are

- We construct an accurate energy-consumption-based OF by considering the Frame Error Ratio (FER) in the physical layer, a limited number of MAC retransmissions (including the first transmission attempt) and the number of hops in the network layer. The achievable throughput is also treated as an important design factor.

- We design a routing algorithm, which employs the energyconsumption-based OF as our design metric. An attractive balance is attained between the energy consumed and the throughput achieved.

The rest of the paper is organized as follows. Our proposed energyconsumption-based OF is detailed in Section II. In Section III, we analyze the achievable performance. Finally, our conclusions are provided in Section IV.

\section{TheoreticAl ANALYsis}

In this treatise we consider the transmit energy consumed by the data packets during their transmission, which are generated from the application layer. The energy consumed by other packets, such as routing and MAC control packets is not considered. In other words, our idealized simplifying assumption is that the energy consumed during the process of route discovery is negligible. The elimination of this simplification was set aside for our future work. The OF we proposed is invoked for making routing-related decisions, which directly influence the energy consumed by future data packets. All nodes are assumed to be stationary. Only a single source-destination 
pair is supported in our network and only a single node has the chance of transmitting in a time slot, once the route was determined. The number of nodes in the network is denoted by $N$, while $H$ indicates the number of hops in an established route. The transmit power of each node assigned to a single transmission attempt is $P_{i}$ and the corresponding energy is $E_{i}$. We assume that all the data packets have the same length and all nodes have the same transmission rate. Hence, if $P_{i}$ is the same at each node, then $E_{i}$ is the same, which may hence be denoted by a constant $E$, while $F E R_{i}$ is the FER of the $i$ th hop in a route that reflects the channel quality information. We opt for using $p_{i}$ as the probability of successful transmission in the $i$ th hop, which is given by $p_{i}=1-F E R_{i}$. The maximum number of MAC retransmission is $N_{r}$, which includes the first transmission attempt and $N_{r}=\infty$ implies having an infinite number of MAC retransmissions. The $\mathrm{OF}$ incorporates the total transmit energy normalized by the number of successfully delivered information bits. The total energy consumption is denoted by $E_{\text {total }}$, while its normalized version is denoted by $\bar{E}_{\text {total }}$. The throughput is defined as the number of information bits successfully delivered to the destination per second, which is $T_{e 2 e}$.

\section{A. Objective Function}

The employment of the OF proposed in $[5,7,8]$ is feasible only when the affordable number of MAC retransmissions is infinite, which is formulated as

$$
E_{\text {total }}=\sum_{1}^{H} \frac{E_{i}}{1-F E R_{i}}
$$

where $\frac{1}{1-F E R_{i}}$ is the expected number of transmission attempts required for successfully delivering a packet across link $i$. As seen from Eq. (1), the total energy of all hops is simply summed, which suggests that the success of the individual links in a route is deemed to be independent of each other, since we assume that an infinite number of MAC retransmissions is affordable.

Naturally, having an infinite number of MAC retransmissions will impose a potentially infinite end-to-end delay at the destination, which is not realistic. In realistic environments, the wireless link may become broken owing to packet errors if the maximum number of MAC retransmissions has been exhausted. A broken link may trigger a route-repair or even route re-discovery for the sake of maintaining the current source-destination communications session. The routerepair is often required at the upper-node's broken link, while the route re-discovery should be initiated by the source. All these actions may consume more energy and naturally they reduce the attainable throughput. Additionally, the success of a specific hop emanating from a node relies on the success of all previous hops. If any of the previous links is broken, then no packet will be forwarded towards the destination. Naturally, any link is more likely to break if the number of MAC retransmissions is limited to $N_{r}$. We divide the energy consumption considered into two parts: the energy consumed by the data packets which succeed in reaching the destination and the energy consumed by the data packets which are dropped before reaching the destination. We define the time slot duration of a single transmission attempt across a given link as $T$. Given the same data packet length and the same transmission rate at each node, $T$ is a constant value. Here, the energy-conscious OF of a two-hop route is detailed as an example. We use $p_{s}$ and $p_{f}$ to denote the probability of a packet being successfully delivered to the destination successfully and being dropped before reaching the destination, respectively. By contrast, the probability $p_{s}(\tau)$ indicates that the packet spends a time duration of $\tau$ successfully propagating from the source to the destination and hence we have:

$$
\begin{aligned}
p_{s}(2 T)= & p_{1} p_{2} \\
p_{s}(3 T)= & \left(1-p_{1}\right) p_{1} p_{2}+p_{1}\left(1-p_{2}\right) p_{2} \\
p_{s}(4 T)= & \left(1-p_{1}\right)^{2} p_{1} p_{2} \\
& +\left(1-p_{1}\right) p_{1}\left(1-p_{2}\right) p_{2}+p_{1}\left(1-p_{2}\right)^{2} p_{2} \\
\vdots & \vdots \\
p_{s}\left(2 N_{r}\right)= & \left(1-p_{1}\right)^{N_{r}-1} p_{1}\left(1-p_{2}\right)^{N_{r}-1} p_{2}
\end{aligned}
$$

Therefore $p_{s}$ is given by

$$
\begin{aligned}
p_{s} & =p_{s}(2 T)+p_{s}(3 T)+p_{s}(4 T)+\cdots+p_{s}\left(2 N_{r}\right), \\
& =\sum_{1}^{N_{r}} \sum_{1}^{N_{r}}\left(1-p_{1}\right)^{i_{1}-1} p_{1}\left(1-p_{2}\right)^{i_{2}-1} p_{2} .
\end{aligned}
$$

Since during a single time slot $T$ the nodes consume an energy of $E$, the estimated total energy $E_{s}$ consumed by a successfully delivered packet in a two-hop route is

$$
\begin{aligned}
E_{s}= & {\left[2 p_{s}(2 T)+3 p_{s}(3 T)+4 p_{s}(4 T)\right.} \\
& \left.+\cdots+2 N_{r} p_{s}\left(2 N_{r}\right)\right] E .
\end{aligned}
$$

In a similar way, the time $T_{s}$ required for a packet, which is successfully delivered from the Source $(S)$ to the Destination $(D)$ is given by

$$
\begin{aligned}
T_{s}= & {\left[2 p_{s}(2 T)+3 p_{s}(3 T)+4 p_{s}(4 T)\right.} \\
& \left.+\cdots+2 N_{r} p_{s}\left(2 N_{r}\right)\right] T .
\end{aligned}
$$

Additionally, the packets, which exhausted the maximum number $N_{r}$ of MAC retransmissions and were finally dropped before reaching $D$ due to poor channel conditions also consume energy. This energy should also be taken into account in the total energy consumption. The probability $p_{f}$ of the two-hop route for a single packet is described as follows:

$$
\begin{aligned}
p_{f}(1) & =\left(1-p_{1}\right)^{N_{r}}, \\
p_{f}(2) & =\left[\left(1-p_{f}(1)\right)\right]\left(1-p_{2}\right)^{N_{r}}, \\
p_{f} & =p_{f}(1)+p_{f}(2),
\end{aligned}
$$

where $p_{f}(h)$ represents the probability of the packet becoming dropped during the $h$ th hop. Therefore, we quantify the energy $E_{f}$ consumed by a dropped packet as follows:

$$
E_{f}=\left[N_{r} p_{f}(1)+\sum_{1}^{N_{r}}\left(1-p_{1}\right)^{i_{1}-1} p_{1}\left(1-p_{2}\right)^{N_{r}}\left(i_{1}+N_{r}\right)\right] E \text {. }
$$

Similarly, the average time $T_{f}$ required by a packet to propagate from $S$ up to the broken link is formulated as

$$
T_{f}=\left[N_{r} p_{f}(1)+\sum_{1}^{N_{r}}\left(1-p_{1}\right)^{i_{1}-1} p_{1}\left(1-p_{2}\right)^{N_{r}}\left(i_{1}+N_{r}\right)\right] T .
$$

Hence, the probability $p_{s}$ and $p_{f}$ for an $H$-hop route are given as follows:

$$
\begin{aligned}
p_{s}= & \sum_{1}^{N_{r}} \cdots \sum_{1}^{N_{r}}\left(1-p_{1}\right)^{i_{1}-1} p_{1}\left(1-p_{2}\right)^{i_{2}-1} p_{2} \\
& \cdots\left(1-p_{H}\right)^{i_{H}-1} p_{H} \\
p_{f}= & p_{f}(1)+\sum_{2}^{H} p_{f}(h), h \neq 1,
\end{aligned}
$$


where

$$
\begin{aligned}
p_{f}(h)= & \sum_{1}^{N_{r}} \cdots \sum_{1}^{N_{r}}\left(1-p_{1}\right)^{i_{1}-1} p_{1} \cdots\left(1-p_{h-1}\right)^{i_{h-1}-1} p_{h-1} \\
& \left(1-p_{h}\right)^{N_{r}}, h \neq 1 .
\end{aligned}
$$

Therefore, the energy $E_{s}$ consumed by a successfully delivered packet and the energy $E_{f}$ consumed by a packet which is dropped before reaching the destination are formulated as:

$$
\begin{aligned}
E_{s}= & {\left[\sum_{1}^{N_{r}} \cdots \sum_{1}^{N_{r}}\left(1-p_{1}\right)^{i_{1}-1} p_{1}\left(1-p_{2}\right)^{i_{2}-1} p_{2}\right.} \\
& \left.\cdots\left(1-p_{H}\right)^{i_{H}-1} p_{H}\left(i_{1}+i_{2}+\cdots+i_{H}\right)\right] E, \\
E_{f}= & {\left[N_{r} p_{f}(1)\right] E+\sum_{2}^{H} E_{f}(h), h \neq 1, }
\end{aligned}
$$

where

$$
\begin{aligned}
E_{f}(h)= & {\left[\sum_{1}^{N_{r}} \cdots \sum_{1}^{N_{r}}\left(1-p_{1}\right)^{i_{1}-1} p_{1} \cdots\left(1-p_{h-1}\right)^{i_{h-1}-1} p_{h-1}\right.} \\
& \left.\left(1-p_{h}\right)^{N_{r}}\left(i_{1}+\cdots+i_{h-1}+N_{r}\right)\right] E, h \neq 1
\end{aligned}
$$

Similarly, we can derive the time $T_{s}$ spent by a successfully delivered packet and the time $T_{f}$ spent by a dropped packet throughout their passage, which is as follows:

$$
\begin{aligned}
T_{s}= & {\left[\sum_{1}^{N_{r}} \cdots \sum_{1}^{N_{r}}\left(1-p_{1}\right)^{i_{1}-1} p_{1}\left(1-p_{2}\right)^{i_{2}-1} p_{2}\right.} \\
& \left.\cdots\left(1-p_{H}\right)^{i_{H}-1} p_{H}\left(i_{1}+i_{2}+\cdots+i_{H}\right)\right] T, \\
T_{f}= & {\left[N_{r} p_{f}(1)\right] T+\sum_{2}^{H} T_{f}(h), h \neq 1, }
\end{aligned}
$$

where

$$
\begin{aligned}
T_{f}(h)= & {\left[\sum_{1}^{N_{r}} \cdots \sum_{1}^{N_{r}}\left(1-p_{1}\right)^{i_{1}-1} p_{1} \cdots\left(1-p_{h-1}\right)^{i_{h-1}-1} p_{h-1}\right.} \\
& \left.\left(1-p_{h}\right)^{N_{r}}\left(i_{1}+\cdots+i_{h-1}+N_{r}\right)\right] T, h \neq 1 .
\end{aligned}
$$

Therefore, the total normalized transmit energy consumption becomes:

$$
\bar{E}_{t o t a l}=\frac{E_{t o t a l}}{p_{s}}=\frac{E_{s}+E_{f}}{p_{s}} .
$$

Similarly, the end-to-end throughput $T_{e 2 e}$ is given as

$$
T_{e 2 e}=\frac{p_{s}}{T_{s}+T_{f}} .
$$

For simplifying Eq. (24) and Eq. (25), we define the mathematical short-hand of $A\left(p_{i}\right)=\frac{1-\left(1-p_{i}\right)^{N_{r}}}{p_{i}}-N_{r}\left(1-p_{i}\right)^{N_{r}}$ and $B\left(p_{i}\right)=1-$ $\left(1-p_{i}\right)^{N_{r}}$, thus we have $p_{s}=\prod_{1}^{H} B\left(p_{i}\right)$. The simplified normalized energy-conscious $\mathrm{OF}$ and end-to-end throughput are formulated in Eq. (26) and Eq. (27) on the top of the next page.

\section{B. Routing Algorithm}

A low-complexity routing algorithm was also designed in this paper based on Dijkstra's shortest-path algorithm [10], which employs the OF of Eq. (26) for making routing decisions. As seen from Eq. (26) and Eq. (27), the normalized energy consumption and the end-to-end throughput are inversely proportional to each other, which is formulated as:

$$
\bar{E}_{t o t a l} \propto \frac{1}{T_{e 2 e}}
$$

Eq. (28) shows that once the route having the lowest estimated normalized energy consumption is chosen to transmit data, the achieved end-to-end throughput would be maximized, if no route-repair and route re-discovery were allowed. Indeed, the energy-conscious OF is capable of reflecting the probability of the route becoming broken because it considers both the FER, as well as the maximum number of MAC retransmission and the number of hops.

The process of route discovery is described in the pseudo-code given by Fig. 1, where $S$ represents the source and $D$ represents the destination. In Fig. $1 E_{S \rightarrow n, t}$ denotes the estimated normalized energy consumption for the route spanning from $S$ to node $n$ at time instant $t$, while $E_{S \rightarrow n}$ is used for storing the minimum normalized energy consumption for every node in every time-slot of duration $T$. The symbol $\leftarrow$ implies assigning the right-hand-side (rhs) value to the left-hand-side (lhs) value, while $H_{\max }$ is the maximum number of hops in Fig. 1. The Route REQuest (RREQ) message of Fig. 1 is a broadcast packet, which is used for finding appropriate routes leading to $D$, while the Route REPly (RREP) message is used for returning all the route identifiers to $S$.

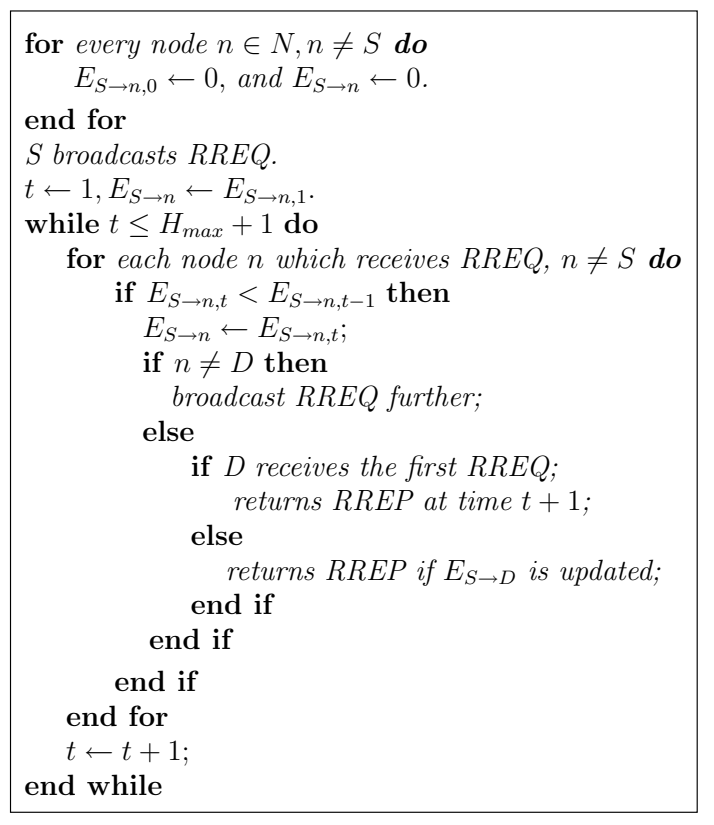

Fig. 1. The process of route discovery in our proposed low-complexity routing algorithm.

Indeed, the RREP message carries the descriptors or identifiers of all the adequate routes to $S$. Once $S$ receives the route-identifiers in the RREP message, it calculates the estimated normalized energy consumption of the routes using the energy-conscious OF. The route imposing the lowest estimated energy consumption will then be chosen to convey the data. If other RREP messages are also returned back to $S, S$ repeats the process of route selection. When the RREP message propagates through the intermediate nodes back to $S$, all the intermediate nodes also update their routing tables for their potential future use. The energy-conscious OF can make the routing decisions more reliable than its conventional counterpart, which assumes having an infinite number of MAC retransmissions, since it considers the non-negligible probability of having broken links in the chosen route. Naturally, the cost of broken links would be very high, if the source had to change to another route or carry out full-scale route rediscovery.

\section{Performance Study}

The analytically estimated normalized energy consumption associated both with an infinite and with $N_{r}$ number of MAC retransmissions was calculated from Eq. (1) and Eq. (26), respectively. 


$$
\begin{gathered}
\bar{E}_{\text {total }}=\frac{\left[N_{r}\left(1-\prod_{1}^{H} B\left(p_{i}\right)\right)+\sum_{1}^{H-1}\left(\frac{A\left(p_{i}\right)}{B\left(p_{i}\right)} \prod_{j=1}^{i} B\left(p_{j}\right)\right)\right] E}{\prod_{1}^{H} B\left(p_{i}\right)}, \\
T_{e 2 e}=\frac{\prod_{1}^{H} B\left(p_{i}\right)}{\left[N_{r}\left(1-\prod_{1}^{H} B\left(p_{i}\right)\right)+\sum_{1}^{H-1}\left(\frac{A\left(p_{i}\right)}{B\left(p_{i}\right)} \prod_{j=1}^{i} B\left(p_{j}\right)\right)\right] T} .
\end{gathered}
$$

The network simulator OMNeT++ [11] was used for generating the simulation results. The analytical and simulation based results will be compared for the sake of quantifying the accuracy of the proposed $\mathrm{OF}$ and to quantify its influence on the routing decisions.

Two simple network topologies were studied. In the first one all the nodes are equi-spaced along a line, as seen in Fig. 2. The distance

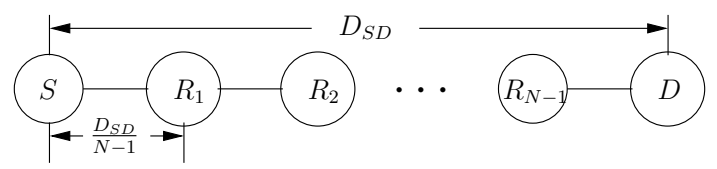

Fig. 2. Test-topology relying on $N$ nodes.

between $S$ and $D$ is $D_{S D}$. Then the distance between two adjacent nodes is $\frac{D_{S D}}{N-1}$. For the sake of fair comparisons, we fixed $D_{S D}$ and varied the number of intermediate nodes between $S$ and $D$. If the transmit power for a two-hop topology is $0.016 \mathrm{~mW}$, which is denoted by $P_{i_{2}}$, then the transmit power for an $(N-1)$-hop topology becomes:

$$
P_{i_{N-1}}=P_{i_{2}}\left(\frac{2}{N-1}\right)^{2}
$$

The normalized energy consumption and the end-to-end throughput evaluated both from Eq. (26) and Eq. (27) as well as by simulations are portrayed in Fig. 3 and Fig. 4. The data packet length is 8688 bits, which does not include the physical layer header. The transmission rate of the nodes is 6 Mbits/s. Fig. 3 displays

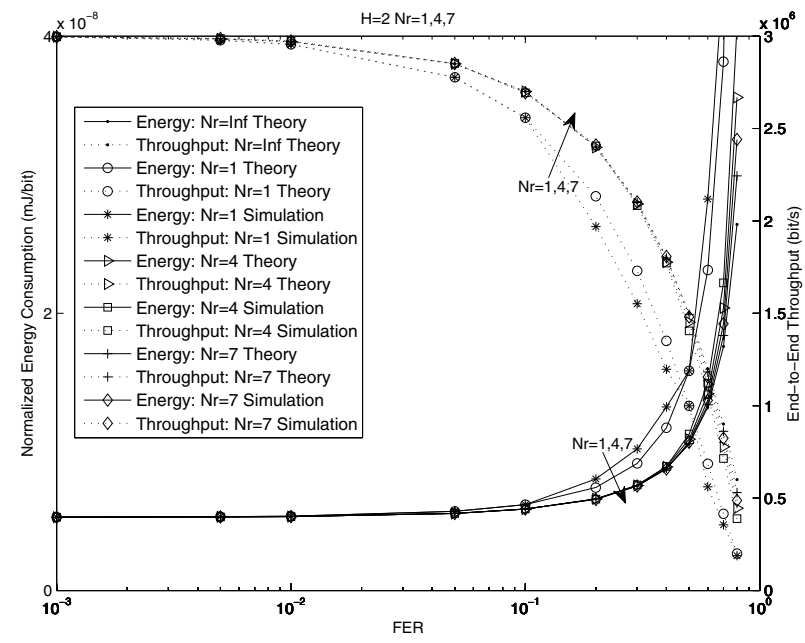

Fig. 3. The normalized energy consumption and the end-to-end throughput versus FER and $N_{r}$.

three groups of performance curves recorded at $N_{r}=1,4$ and 7 , respectively, for both the normalized energy consumption and for the end-to-end throughput. The performance figures recorded for the infinite number of MAC retransmissions scenario, namely for $N_{r}=\infty$ are identical for the theory evaluated from Eq. (1) and for our simulations. All the analytical and the simulation based

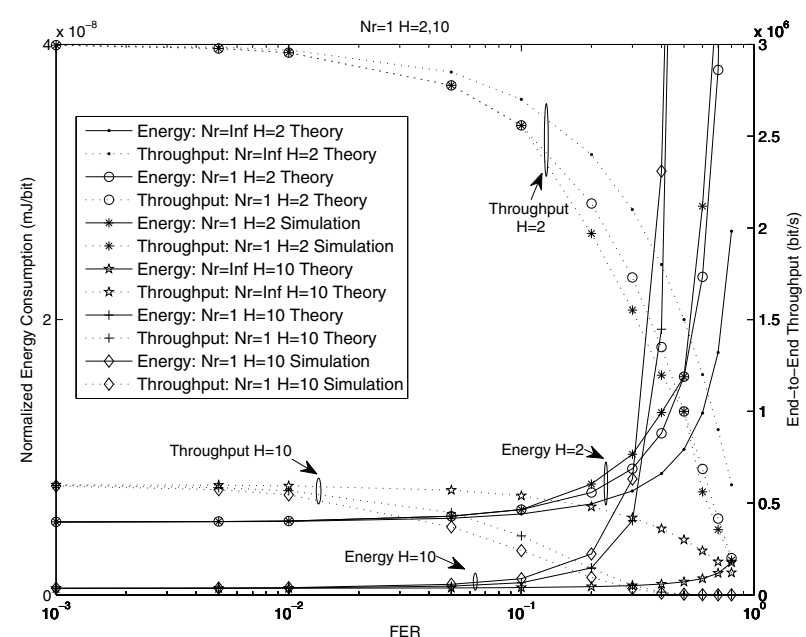

Fig. 4. The normalized energy consumption and the end-to-end throughput versus FER and $H$.

values recorded for the normalized energy consumption increase, when the FER increases. By contrast, the curves representing the end-to-end throughput decrease, when the FER increases. The reason for this observation is that a high FER in a link indicates a high breakage probability not only for the specific link and but also for the entire route, when retransmissions are required. However, if $N_{r}$ is sufficiently high, then the success probability of a packet across a link or even the entire route becomes higher. This trend is presented in Fig. 3, where the curve recorded for $N_{r}=7$ is seen to be close to that of $N_{r}=\infty$. The discrepancy between the theoretical value and the simulation-based value becomes higher when $N_{r}$ is reduced and simultaneously the FER is increased. Fig. 3 also shows that the theoretical energy consumption of Eq. (26) based on our energyconscious OF is closer to the simulation based values than those based on the OF relying on an infinite number of MAC retransmissions. Naturally, the advantage of the proposed OF is more substantial for $N_{r}=1$. Fig. 4 also displays two groups of performance curves, one group for the normalized energy consumption and the other group for the end-to-end throughput, which are associated with $H=2$ and 10 , respectively. When $H$ is increased, the normalized energy consumption is reduced and the end-to-end throughput is decreased, because the distance between a pair of adjacent nodes is reduced and therefore the transmit power required at each node for successfully delivering a packet is reduced, as suggested by Eq. (29). Similarly, the theoretical values estimated based on the proposed OF are closer to the simulated values than to those estimated on the basis of an infinite number of MAC retransmissions, especially when both $H$ and the FER are high. Hence we conclude from Fig. 3 and Fig. 4 that our proposed energy-conscious $\mathrm{OF}$ is more accurate than the one assuming an infinite number of MAC retransmissions at high FERs, or for a high number of hops at a low maximum number of MAC retransmissions.

Let us now consider the six-node topology, seen in Fig. 5. The line 
between each pair of nodes indicates that they are within the sensing range of each other. Node $A$ is the source and $F$ is the destination. All the nodes have the same transmit power of $0.016 \mathrm{~mW}$. The FER is assumed to be a random variable for each link which is generated by a random generator and uniformly distributed in [0,1]. All the other parameters are the same as in the linear topology of Fig. 2.

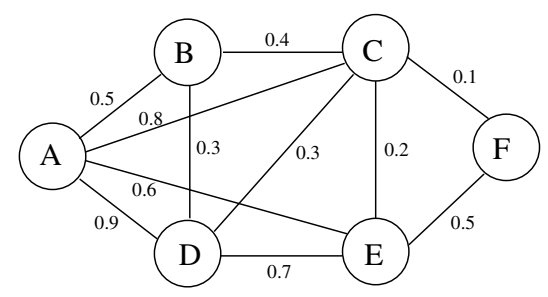

Fig. 5. A simple six-node network topology.

Assuming a specific group of FERs between every pair of nodes for example, the matrix $\mathbf{M}_{F E R}$ of FER is given by

$$
\mathbf{M}_{F E R}=\left[\begin{array}{llllll}
0 & 0.5 & 0.8 & 0.9 & 0.6 & 1.0 \\
0.5 & 0 & 0.4 & 0.3 & 1.0 & 1.0 \\
0.8 & 0.4 & 0 & 0.3 & 0.2 & 0.1 \\
0.9 & 0.3 & 0.3 & 0 & 0.7 & 1.0 \\
0.6 & 1.0 & 0.2 & 0.7 & 0 & 0.5 \\
1.0 & 1.0 & 0.1 & 1.0 & 0.5 & 0
\end{array}\right]
$$

where each row and column corresponds to a specific node. As seen from Fig. 6, when $N_{r}$ increases, the normalized energy consumption decreases and the end-to-end throughput increases. Table I portrays the routes selected when using either the conventional OF relying on an infinite number of MAC retransmissions or our proposed OF. Columns 'Energy1' and 'Energy2' show the simulation based values of the normalized energy consumption, when choosing the routes 'A-E-F' and 'A-E-C-F', respectively. We can see that the algorithm assuming an infinite number of MAC retransmissions always chooses the route 'A-E-F', while our proposed algorithm opts for 'A-E-C-F', when $N_{r}=1,2$ and chooses 'A-E-F' for $N_{r}=3$ to 7 . By observing the normalized energy consumptions listed in columns 'Energy1' and 'Energy2', our proposed algorithm is seen to make the right decisions, which saves energy and improves the throughput attained. By observing that the route 'A-E-C-F' is selected for $N_{r}=1,2$, the route associated with the minimum number of hops does not always perform well, when considering the attainable energy efficiency.

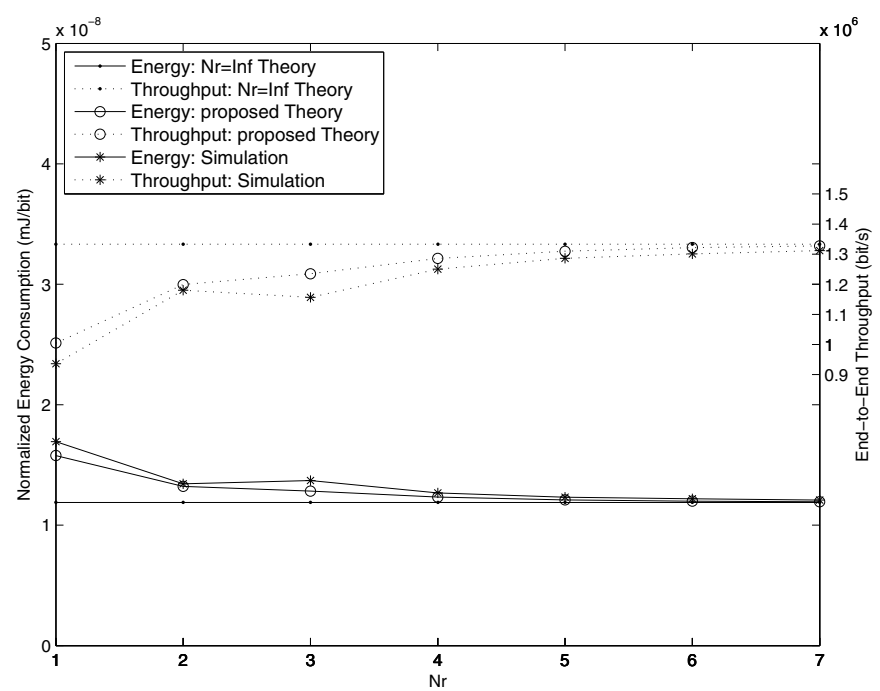

Fig. 6. The normalized energy consumption and the end-to-end throughput of the six-node topology of Fig. 5.
TABLE I

ROUTE SELECTION

\begin{tabular}{|l|l|l|l|l|}
\hline$N_{r}$ & $\begin{array}{l}N r=\infty \\
\text { Theory }\end{array}$ & $\begin{array}{l}\text { Proposed } \\
\text { Theory }\end{array}$ & $\begin{array}{l}\text { Energy1(mJ/bit }) \\
\text { A-E-F }\end{array}$ & $\begin{array}{l}\text { Energy2(mJ/bit }) \\
\text { A-E-C-F }\end{array}$ \\
\hline \hline 1 & A-E-F & A-E-C-F & $2.1025 \mathrm{e}-08$ & $1.6934 \mathrm{e}-08$ \\
\hline 2 & A-E-F & A-E-C-F & $1.5369 \mathrm{e}-08$ & $1.3428 \mathrm{e}-08$ \\
\hline 3 & A-E-F & A-E-F & $1.3705 \mathrm{e}-08$ & $1.5506 \mathrm{e}-08$ \\
\hline$\cdots$ & $\cdots$ & $\cdots$ & $\cdots$ & $\cdots$ \\
\hline
\end{tabular}

\section{CONCLUSIONS}

An energy-conscious OF was proposed, which is takes into account the FER of the physical layer, a finite number of $N_{r}$ MAC retransmissions and the actual number of hops encountered in the network layer. Unlike the previously proposed OFs, which usually assume that the number of MAC retransmissions is infinite, the proposed OF represents the real-world scenarios more closely, since it assumes a finite number of $N_{r}$ MAC retransmissions. As a result, the simulation results confirm the theoretical results relying on our proposed $\mathrm{OF}$ more closely, especially when the FER is high, the number of maximum MAC retransmissions is low and the number of hops is high. A low-complexity routing algorithm was also designed for making reliable routing decisions based on the energy consumption using our energy-conscious OF, which allowed us to minimize the normalized energy consumption without unduly compromising the end-to-end throughput.

\section{REFERENCES}

[1] A. Ibrahim, H. Zhu, and K. J. R. Liu, "Distributed energy-efficient cooperative routing in wireless networks," IEEE Transactions on Wireless Communications, vol. 7, no. 10, pp. 3930 -3941, Oct. 2008.

[2] E. Baccarelli, M. Biagi, C. Pelizzoni, and N. Cordeschi, "Maximum-rate node selection for power-limited multiantenna relay backbones," IEEE Transactions on Mobile Computing, vol. 8, no. 6, pp. 807 -820, June 2009.

[3] M. Sikora, J. N. Laneman, M. Haenggi, D. J. Costello, and T. E. Fuja, "Bandwidth- and power-efficient routing in linear wireless networks," IEEE Transactions on Information Theory, vol. 52, no. 6, pp. $2624-$ 2633, June 2006.

[4] C. Bae and W. E. Stark, "End-to-end energy-bandwidth tradeoff in multihop wireless networks," IEEE Transactions on Information Theory, vol. 55, no. 9, pp. $4051-4066$, Sept. 2009.

[5] S. Banerjee and A. Misra, "Minimum energy paths for reliable communication in multi-hop wireless networks," in MobiHoc '02 Proceedings of the 3rd ACM international symposium on mobile ad hoc networking and computing, Lausanne, Switzerland, June 2002, pp. 146-156.

[6] S. Cui, R. Madan, A. J. Goldsmith, and S. Lall, "Cross-layer energy and delay optimization in small-scale sensor networks," IEEE Transactions on Wireless Communications, vol. 6, no. 10, pp. 3688 -3699, Oct. 2007.

[7] Q. F. Dong and S. Banerjee, "Minimum energy reliable paths using unreliable wireless links," in MobiHoc '05 Proceedings of the 6th ACM international symposium on mobile ad hoc networking and computing, Urbana-Champaign, Illinois, USA, May 2005, pp. 449-459.

[8] J. Zhu, C. Qiao, and X. Wang, "On accurate energy consumption models for wireless ad hoc networks," IEEE Transactions on Wireless Communications, vol. 5, no. 11, pp. 3077 -3086, November 2006.

[9] X.-Y. Li, Y. Wang, H. Chen, X. Chu, Y. Wu, and Y. Qi, "Reliable and energy-efficient routing for static wireless ad hoc networks with unreliable links," IEEE Transactions on Parallel and Distributed Systems, vol. 20, no. 10, pp. $1408-1421$, Oct. 2009.

[10] D. Bertsekas and R. Gallager, Data Networks. Upper Saddle River, New Jersey, USA: Prentice Hall, 1992.

[11] A. Varga and R. Hornig, "An overview of the OMNeT++ simulation environment," in Proc. 1st international conference on simulation tools and techniques for communications, networks and systems \& workshops Simutools '08, 2008, pp. 1-10. 